\title{
Meeting the need for COVID-19 models
}

\author{
Over a year into the pandemic, animal providers have been working to meet changing demand for the animal \\ models needed to study SARS-CoV-2
}

\section{Ellen P. Neff}

\begin{abstract}
t didn't take long for the research community to take note of the emerging threat from SARS-CoV-2, the virus behind the pandemic that's been ongoing for over a year now. While many labs and institutions were forced to put work on hold and adjust to distancing restrictions that limited personnel capacity, teams that could make the pivot - those working at the BSL3-capable facilities required to work with an airborne infectious agent such as SARS-CoV-2 - quickly shifted to study the virus. Animal providers have been working to keep up with the continued, and changing, demand.
\end{abstract}

\section{Speeding up small options}

Fortunately, SARS-CoV-2 shares similarities with related coronaviruses that were responsible for prior outbreaks of diseases, including Severe Acute Respiratory Syndrome (SARS) and Middle Eastern Respiratory Syndrome (MERS). Small animal models developed for studying those viruses, including ferrets, golden hamsters, and a number of mouse models, have given researchers and pharmaceutical companies a helpful starting point.

Golden hamsters, which are more naturally susceptible to the virus than their murine relatives and have been used throughout the pandemic to study therapeutics and vaccines as well as more mechanistic details about SARS-CoV-2, quickly came into high - and continued demand. "In response to this, we expanded our capacity for breeding," says Steve Festin, Director of Scientific and Commercial Development at preclinical model and services provider Charles River, one of the commercial providers of golden hamsters with colonies in both the US and China.

Hamsters have been valuable because their angiotensin-converting enzyme 2 (ACE2) protein is similar to that of humans; ACE2 is a main receptor that the coronavirus uses to gain entry into a host's cells. The structure of mouse ACE2 is different enough that it doesn't interact with the virus in the same way, so mice are not naturally susceptible to infections (although there are some mouse-adapted coronavirus strains, and a recent preprint published in bioRxiv suggests some emerging viral variants of SARS-CoV-2 can naturally infect mice). Nevertheless, mice are playing pivotal roles in understanding, treating, and preventing COVID-19. For the providers of these animals, it was off to their portfolios and in some cases, freezers.

At mouse provider Taconic, they decided at the start of the pandemic to pull an Ace 2 knockout mouse out of cryopreservation to scale up to live production again; that mouse had been developed by Lexicon Pharmaceuticals for studying Ace2's role in lung failure during the SARS-CoV outbreak in the mid-2000s. "That was a bet that we hedged that ended up being useful for the vaccine community," says Terina Martinez, a field application scientist with Taconic. They've also started increasing the available capacity of existing transgenic human leukocyte antigen (HLA) mice - these animals are also important for studying the immunogenicity of vaccines, she says.

At the Jackson Laboratory, they started working early last year to increase breeding of a transgenic mouse expressing the human ACE2 gene that had been developed for SARS research by Stanley Perlman's lab at the University of Iowa. "That has probably been one of the biggest mouse models that we've distributed over the last year," says Cat Lutz, director of the Jackson Laboratory's Mouse Repository.

Initial research efforts focused heavily on treatment and vaccine development. Perlman's hACE2 mice, referred to as K18s, for example, develop severe phenotypes and die within about a week following infection, says Lutz - any extension in lifespan provides a relatively quick and efficient readout for whether or not an intervention is helping. As the pandemic has worn on, focus has shifted towards providing models to better understand the disease itself, its long-term ramifications, and the consequences of all those emerging viral variants.

With collaborators the Jackson Laboratory has been working on a number of new mouse models; these efforts have included placing hACE2 under different tissue-specific promotors, creating mice with single copies of hACE2 in the mouse Ace 2 locus as well as substituting key amino acids for targeted 'humanization of other loci, and crossing K18 mice, originally on a C57BL/6 genetic background, into additional mouse strains to see how these different variables might influence disease phenotypes. They've also been exploring a mouse adapted coronavirus, passaged through susceptible $\mathrm{BALB} / \mathrm{c}$ mice, in non-transgenic animals, which enables mapping and genetic tracing of susceptibility and resilience features.

Taconic has been scaling up production of a handful of different genetically modified mice that express human proteins. These include several hACE2 lines that were developed at Chien-Te Tseng's lab at the University of Texas Medical Branch the for the first SARS, as well as Dipeptidyl peptidase 4 (dpp4) knockout and humanized DPP4vlines that Tseng's lab made to study MERS (DPP4 is a coronavirus co-receptor that SARS-CoV-2 may also take advantage of to enter host cells). The company is also collaborating with the National Emerging Infectious Disease Laboratories at Boston University for input on model design strategies and for validation of genetic models on Taconic's B6 and super-immunodeficient backgrounds. The company plans to release any that show utility during 2021. "We don't know what biology at the gene level will translate to biology at the immune level, but we're taking a shots-on-goal approach," Martinez says, noting they continue to engage with the community, directly and via emerging literature, to try and anticipate changes in demand.

Charles River has also been partnering with research labs to develop and validate genetic models that express human ACE2. The company is also working on models engrafted with different human immune cells; one example in the HuNCG model, an immunodeficient mouse engrafted with human CD34+ cells. Humanizing the mice in various ways increases their susceptibility to the virus and thus relevance to human disease.

Developing and producing these animals does take time - about three months, minimum, although genetically modified animals can take between nine months and a year. "We made decisions in March [last year] 
that we couldn't even look at until June or July," says Nadia Rosenthal, Scientific Director of the Jackson Laboratory

Increasing production of live colonies that are ready to ship on demand comes with constraints. "We can't just turn on and off animal production like a switch," says Martinez. "It is still a biological process with rate limited steps." Even with advanced reproductive technologies and strategies for rapid expansion, it still takes time for the animals to reproduce and for those pups to grow up and be used to breed another generation, or be sent out to a study. "You have to reserve breeders in order to be able to produce more, so it's a bit of a Catch-22," says Festin.

There are ethical considerations to be mindful of too. "We're not going to overproduce is we don't think there's going to be a use for them in research. We've been both intelligent and lucky in striking the right balance there," says Martinez. "For the ones that are in development, we can't get them on the shelf fast enough."

\section{The primate problem}

Those working with nonhuman primates, animals with genetics and immune systems that more closely resemble those of humans, were also quick to pivot their attention to the emerging pandemic.

As early as February 2020, researchers at the Biomedical Primate Research Center (BPRC) and Erasmus Medical Center in the Netherlands were already preparing to study the novel variant in cynomolgus macaques; that work was posted as a preprint in March and published in Science online in April.

At the BRPC and the handful of research institutes around the world that maintain breeding colonies of primates, COVID-19 quickly took top priority. On-going studies continued - the animals are too valuable for their studies to be cut short - but new projects that hadn't yet started were put on hold for time (though since resumed) as animals were re-directed to studying SARS-CoV-2, says Joyce Cohen, the Associate Director of the Division of Animal Resources at the Yerkes National Primate Research Center (NPRC), one of seven such facilities funded by the National Institutes of Health (NIH) in the United States.

At contract research organizations providing primates, focus shifted as well. Joachim Confais, the Chief Science Officer for Cynbiose in France, notes postponements from study sponsors early on the pandemic as attention moved to COVID-19. Cynbiose provides mostly cynomolgus macaques, along with other species including African Green monkeys and marmosts, to both academic researchers and pharma and biotech companies across Europe as well as to some US-based companies; last spring, they began collaborating with the VirPath laboratory at Claude Bernard Lyon University to develop an African Green COVID-19 model as an alternative to macaques. The company had been planning an expansion of their primate capacity prior to the pandemic, including construction of BSL- 3 containment facilities for on-site testing; COVID-19 has been hurrying that along, Confais says.

As with rodents, much of the initial focus with nonhuman primates was on treatment and prevention; that has similarly shifted to exploring the mechanisms underlying the disease and virus transmission, while continuing to test vaccines and treatments against new variants. 'Normal' workloads are returning, but it's not the end of COVID research with the animals by any means.

Jans Langermans, deputy director of the BPRC, expects COVID-19 will remain a priority for at least another year and a half "There will be a post-COVID time, but this was a warning," says Langermans. With still much to learn about SARS-CoV-2, as well as other emerging infectious diseases - novel influenzas, Zika, chikungunya and Ebola, to name just a few -the need for primates is not soon to run out.

The demand, however, has been outstripping the supply - a problem that was emerging before COVID-19 took center stage. The US had been discussing the issue for several years and released a report in 2018 about a potential domestic shortage. In Europe, winds have been shifting; in 2018, the Dutch government for example passed a motion that the BPRC colony was to be dropped by about 1,500 animals to just a thousand and the number of studies with the primates limited to 150 per year.

When early last year, just as the scale of the pandemic was becoming apparent, the Chinese government banned exports of nonhuman primates, researchers without their own breeding programs found themselves in need. "We got an enormous amount of requests for animals," says Langermans. "Of course, we didn't have them - we needed them for our own research."

Confais calls the export ban the cherry on top of changing primate demand Although most of the animals imported into Europe don't come from China - rather, many come from other countries in Southeast Asia as well as Mauritius - the ban has nevertheless put extra strain on those providers. "It's forced us to organize longer term," says Confais. "Now we really have to plan our supply."

In the US, the NIH decided to prioritize the animals to NIH-funded work, organized through the Accelerating COVID-19 Therapeutic Interventions and Vaccines (ACTIV) initiative. "So far, we have been able to find animals to do the COVID studies that we need, at least from the NPRC perspective," says Cohen, though the Centers are taking steps to minimize animal use, for example by sharing data from animals across the enters as controls for experiments. But, she says, "we have all certainly seen domestic researchers turning to us and looking for additional animals because they could no longer get their supply like they had before."

At a recent COVID-19 workshop held by the US National Academies of Sciences, Engineering and Medicine, Gale Galland from the US CDC Quarantine and Border Health Services Branch, which oversees animal importation into the US, noted that in 2019 over 20,000 animals came in from China; in 2020, only 3,723 made it in before the ban took effect. The number since has been zero, leaving those in need to look to other countries in Southeast Asia as well as the Caribbean to supply animals.

Commercial providers are exploring how they might help make up the difference. "We're getting calls 24/7," says Greg Westergaard, the CEO of Alpha Genesis, a commercial provider of rhesus and cynomolgus macaques based in South Carolina. The Alpha Genesis colony can currently house about 6,000 animals, and the company has sped up pre-pandemic plans to expand to $10-12,000$ animals as domestic shortages continue. "I don't think there's any way around it, because the monkeys are so critical," he says.

Expansions, however, take time, money and considerable infrastructure. There needs to be holding as well as medical space, plus staff to care for an increasing number of animals. Gestation in macaques takes about six months, and not all females will become pregnant in a given year; afterwards, most animals won't be ready to go on to studies for an additional two to three years. As with other animals, reserving females for breeding means those animals can't be used for research, involving a balancing act between expanding colonies and getting research data.

"It's going to be difficult to identify animals, and I think it's going to be a long time before we're fully back to normal," says Cohen.

Ellen P. Neff $\bowtie$
Lab Animal.
$\bigotimes_{e-\text {-mail: ellen.neff@us.nature.com }}$

Published online: 21 April 2021

https://doi.org/10.1038/s41684-021-00759-2 